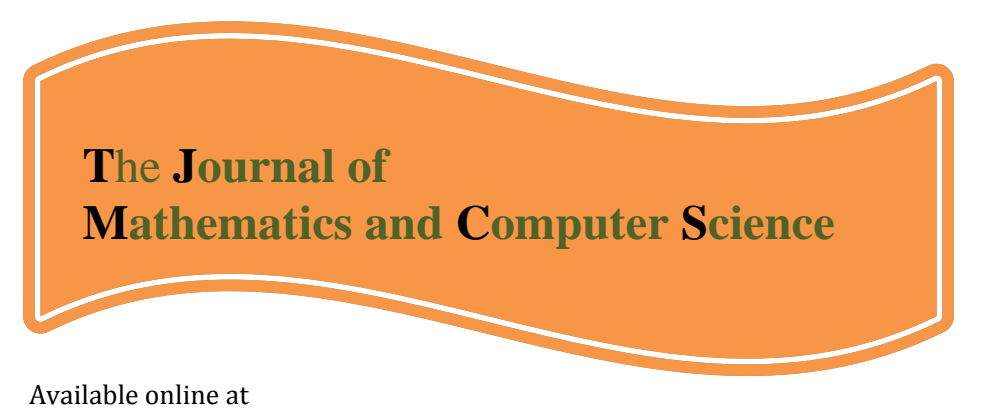

http://www.TIMCS.com

The Journal of Mathematics and Computer Science Vol .2 No.2 (2011) 376-387

\title{
Exact travelling solutions for the sixth-order Boussinesq equation
}

\author{
M. Hosseini ${ }^{{ }^{*}}$, H. Abdollahzadeh ${ }^{2}$, M.Abdollahzadeh ${ }^{\mathbf{3}}$ \\ ${ }^{1}$ Department of Mechanical Engineering, Islamic Azad University, Ghaemshahr, Iran. \\ ${ }^{2}$ University of Kurdistan, Department of Industrial Engineering, Sanandaj, Iran. \\ ${ }^{3}$ Babol University of Technology, Department of Mechanical Engineering P. O. Box: 484, Babol, Iran
}

Received: September 2010, Revised: November 2010

Online Publication: January 2011

\begin{abstract}
In this paper, we establish some distinct exact solutions for a nonlinear evolution equation. The sin-cosine method and the rational Exp-Function and the rational hyperbolic function method are used to construct the solitary travelling wave solutions of the sixth-order Boussinesq equation. These solutions may be important of significance for the explanation of some practical physical problem.
\end{abstract}

Keywords: Traveling wave solutions; sin-cosine method; Exponential rational function method; the rational hyperbolic functions methods, the sixth-order Boussinesq equation

\section{1- Introduction}

Nonlinear evolution equations are widely used as models to describe complex physical phenomena and have a significant role in several scientific and engineering fields. These equations appear in solid state physics [1], fluid mechanics [2], chemical kinetics [3], plasma physics [4], population models, nonlinear optics, propagation of fluxons in Josephson junctions and etc... Analytical exact solutions to nonlinear partial

\footnotetext{
* Corresponding author:

E-mail:Amirhossini_54@yahoo.com
} 
differential equation play an important role in nonlinear science, since they can provide us much physical information and more inside into the physical aspects of the problem and thus lead to further applications. In recent years, quite a few methods for obtaining explicit travelling and solitary wave solutions of nonlinear evolutions equations have been proposed. A variety of powerful methods, such as inverse scattering method [5,6], bilinear transformation [7], Bäcklund and Darboux transformation [7-11], the tanh-sech method [12,13,14], extended tanh method [15], Exp-function method[16-19], the sinecosine method [20-22], the Jacobi elliptic function method [23-25], (G'/G)-expansion method [26,27], Li group analysis[28], He's variational iteration method[29], He's homotopy perturbation method[30-32] and homogeneous balance method [33,34] and so on.

The sine-cosine method was developed by Wazwaz [22] and was successfully applied to nonlinear evolution equations [21,35,36,37], to nonlinear equations systems [38].

In this paper we will apply the sine-cosine method, rational exponential function method and rational hyperbolic function method to obtain the exact traveling wave solution of the sixth-order Boussinesq equation

$u_{t t}-u_{x x}-\left[15 u u_{4 x}+30 u_{x} u_{3 x}+15\left(u_{2 x}\right)^{2}+45 u^{2} u_{2 x}+90 u u_{x}^{2}+u_{6 x}\right]=0$,

Which was recently derived by Wazwaz by generalizing the bilinear forms of the standard Boussinesq equation [39]. Wazwaz obtained the single soliton solutions of the sixth-order Boussinesq equation using the tanh method and multiple-soliton solutions using Hirota bilinear method.

\section{2- Sine-cosine method}

Wazwaz has summarized the main steps introduced for using sine-cosine method, as following:

1- We introduce the wave variables $\xi=x-c t$ into the PDE, we get

$\phi\left(u, u_{t}, u_{x}, u_{t t}, u_{x x}, u_{x t}, u_{x x x} \ldots\right)=0$.

where $u(x, t)$ is travelling wave solution. This enables us to use the following changes:

$u(x, t)=U(\xi)$,

$$
\begin{array}{lrl}
\frac{\partial}{\partial t}=-c \frac{d}{d \xi}, & \frac{\partial^{2}}{\partial t^{2}}=c^{2} \frac{d^{2}}{d \xi^{2}}, \\
\frac{\partial}{\partial x}=\frac{d}{d \xi}, & \frac{\partial^{2}}{\partial x^{2}}=\frac{d^{2}}{d \xi^{2}}, \ldots
\end{array}
$$

And so on for the other derivates. Using (2.3) and(2.1), the nonlinear PDE (2.1))changes to a nonlinear ODE:

$\psi\left(U,-c U^{\prime}, U^{\prime}, c^{2} U^{\prime \prime}, U^{\prime \prime},-c U^{\prime}, U^{\prime \prime \prime} \ldots\right)=0$,

2-If all terms of the resulting ODE contain derivatives in $\xi$, then by integrating this equation, by considering the constant of integration to be zero, we obtain a simplified ODE.

3- By virtue of the technique of solution, we introduce the anstaz:

$$
U(\xi)=u(x, t)=\lambda \sin ^{\beta}(\mu \xi), \quad|\mu \xi|<\frac{\pi}{2}
$$


Or

$$
U(\xi)=u(x, t)=\lambda \cos ^{\beta}(\mu \xi), \quad|\mu \xi|<\frac{\pi}{2 \mu}
$$

Where $\lambda, \mu$ and $\beta$ are parameters are to be determined later, $\mu$ and $c$ are the wave number and the wave speed, respectively, we use:

$$
\begin{aligned}
U(\xi)= & \lambda \sin ^{\beta}(\mu \xi), \\
U^{n}(\xi)= & \lambda^{n} \sin ^{n \beta}(\mu \xi), \\
\left(U^{n}\right)_{\xi}= & n \mu \beta \lambda^{n} \cos (\mu \xi) \sin ^{n \beta-1}(\mu \xi), \\
\left(U^{n}\right)_{\xi \xi}= & -n^{2} \mu^{2} \beta^{2} \lambda^{n} \sin ^{n \beta}(\mu \xi)+n \mu^{2} \lambda^{n} \beta(n \beta-1) \sin ^{n \beta-2}(\mu \xi), \\
\left(U^{n}\right)_{\xi \xi \xi}= & n \lambda^{n} \mu^{3} \beta\left(n \beta^{2}-3 \beta+2\right) \sin ^{n \beta-3}(\mu \xi) \cos ^{3}(\mu \xi) \\
& +\lambda^{n} \mu^{3} n \beta(3 \beta n-2) \sin ^{n \beta-1}(\mu \xi) \cos (\mu \xi),
\end{aligned}
$$

and the derivatives of Eq. (2.6) becomes:

$$
\begin{aligned}
U(\xi)= & \lambda \cos ^{\beta}(\mu \xi), \\
U^{n}(\xi)= & \lambda^{n} \cos ^{n \beta}(\mu \xi), \\
\left(U^{n}\right)_{\xi}= & -n \mu \beta \lambda^{n} \sin (\mu \xi) \cos ^{n \beta-1}(\mu \xi), \\
\left(U^{n}\right)_{\xi \xi}= & -n^{2} \mu^{2} \beta^{2} \lambda^{n} \cos ^{n \beta}(\mu \xi)+n \mu^{2} \lambda^{n} \beta(n \beta-1) \cos ^{n \beta-2}(\mu \xi), \\
\left(U^{n}\right)_{\xi \xi \xi} & =n \lambda^{n} \mu^{3} \beta\left(-n^{2} \beta^{2}+3 n \beta-2\right) \cos ^{n \beta-3}(\mu \xi) \sin ^{3}(\mu \xi) \\
& +\lambda^{n} \mu^{3} n \beta(3 \beta n-2) \sin ^{n \beta-1}(\mu \xi) \sin (\mu \xi),
\end{aligned}
$$

and so on for the other derivatives.

4- We substitute Eq. (2.7) or (2.8) into the reduced equation obtained above in (2.4), balance the terms of the cosine functions when (2.8) is used, or balance the terms of the sine functions when (2.7) is used, and solving the resulting system of algebraic equations by using the computerized symbolic calculations. We next collect all terms with same power in $\cos ^{k}(\mu \xi)$ or $\sin ^{k}(\mu \xi)$ and set to zero their coefficients to get a system of algebraic equations among the unknowns $\mu, \beta$ and $\lambda$. We obtained all possible value of the parameters $\mu, \beta$ and $\lambda$.

\section{The rational function in $\exp (\xi)$ method}

The exp-function method was first proposed by $\mathrm{He}$ and $\mathrm{Wu}$ in 2006[17] and systematically studied in [18,19], and was successfully applied to KdV equation with variable coefficients [40], to high-dimensional nonlinear evolution equation [41], to burgers and combine KdV-mKdV (extended KdV) [42] equations, etc. In this section, we shall seek a rational function type of solution for a given partial equation, in terms of $\exp (\xi)$, of the following form [43]:

$$
U=\sum_{k=0}^{m} \frac{a_{k}}{\left(1+e^{\xi}\right)^{k}}
$$


Where $a_{0}, a_{1}, \ldots, a_{m}$ are some constants to be determined from the solution of (2.4).

Differentiating (3.1) with respect to $\xi$, introducing the result into Eq. (2.4), and setting the coefficients of the same power of $e^{\xi}$ equal to zero, we obtain algebraic equations. The rational function solution of the equation (2.1) can be solved by obtaining $a_{0}, a_{1}, \ldots, a_{m}$ from this system.

\section{The rational hyperbolic functions methods}

The rational hyperbolic functions methods $[44,45]$ can be expressed in the form

$$
U(\xi)=\frac{a_{0}+b_{0} f^{n}(\mu \xi)}{1+a_{1} f^{n}(\mu \xi)}, \quad \xi=x-c t, n=1,2,
$$

Where $f(\mu \xi)$ takes anyone of the hyperbolic functions and $a_{0}, a_{1}, b_{0}, c$ and $\mu$ are parameters that will be determined. The rational hyperbolic functions methods can be applied directly as assumed before. We then collect the coefficients of the resulting hyperbolic functions and setting it equal to zero, and solving the resulting equations to determine the parameters $a_{0}, a_{1}, b_{0}, c$ and $\mu$.

\section{Exact travelling solutions for the sixth-order Boussinesq equation}

\subsection{Application of Sine-cosine method}

As described in Section 2, we make the transformation:

$$
u(x, t)=U(\xi), \quad \xi=x-c t,
$$

Substituting Eq. (5.1) into (1.1) yields an ODE:

$c^{2} U^{\prime \prime}-U^{\prime \prime}-15 U U^{(4)}-30 U^{\prime} U^{\prime \prime \prime}-15 U^{\prime \prime 2}-45 U^{2} U^{\prime \prime}-90 U U^{\prime 2}-U^{(6)}=0$,

Integrating (5.2) twice and using the constants of integration to be zero, we find:

$c^{2} U-U-15 U U^{\prime \prime}-15 U^{3}-U^{(4)}=0$,

Substituting Eq. (2.6) and Eq. (2.8) into (3.3) and rewriting the equation in terms of the sine function gives:

$$
\begin{aligned}
& \left(-\lambda \beta^{4} \mu^{4}-\lambda+c^{2} \lambda\right) \sin ^{\beta}(\mu \xi)+15 \lambda^{2} \beta^{2} \mu^{2} \sin ^{2 \beta}(\mu \xi)-15 \lambda^{3} \sin ^{3 \beta}(\mu \xi) \\
& +\left(-4 \lambda \beta \mu^{4}+8 \lambda \beta^{2} \mu^{4}-6 \lambda \beta^{3} \mu^{4}+2 \lambda \beta^{4} \mu^{4}\right) \sin ^{\beta-2}(\mu \xi) \\
& +\left(6 \lambda \beta \mu^{4}-\lambda \beta^{4} \mu^{4}-11 \lambda \beta^{2} \mu^{4}+6 \lambda \beta^{3} \mu^{4}\right) \sin ^{\beta-4}(\mu \xi) \\
& +\left(15 \lambda^{2} \beta \mu^{2}-15 \lambda^{2} \beta^{2} \mu^{2}\right) \sin ^{2 \beta-2}(\mu \xi)=0
\end{aligned}
$$

Balancing the terms of the sine functions, we have:

$$
\begin{aligned}
& \beta(\beta-1)(\beta-2)(\beta-3) \neq 0, \\
& \beta-4=3 \beta \quad \Rightarrow \beta=-2,
\end{aligned}
$$

Substituting Eq. (5.5b) into Eq.(5.4) and Equating the exponents and the coefficients of each pair of the cosine function, we obtain a system of algebraic equations:

$$
\begin{aligned}
& \sin ^{-6}(\mu \xi):-90 \lambda^{2} \mu^{2}-120 \lambda \mu^{4}-15 \lambda^{3}=0, \\
& \sin ^{-4}(\mu \xi): 60 \lambda \mu^{2}\left(\lambda+2 \mu^{2}\right)=0
\end{aligned}
$$


$\sin ^{-4}(\mu \xi): \lambda\left(-16 \mu^{4}+c^{2}-1\right)=0$

Solving (5.6a)-(5.6c) with the aid of Maple, we obtain:

$$
\lambda=-2 \mu^{2}, \quad c=\sqrt{1+16 \mu^{4}}
$$

The results in Eq. (5.7) can be easily obtained if we also use the sine method (2.5).

Combining (5.7) with (2.6), the following solutions will be obtained:

$$
u_{1}(x, t)=-\frac{2 \mu^{2}}{\cos ^{2}\left(\mu\left(x-\sqrt{1+16 \mu^{4}} t\right)\right)},
$$

Eq.(4.8) will satisfy Eq.(1.1).

\subsection{Application of rational exponential function method}

Now we shall seek a rational function type of solution to the sixth-order Boussinesq equation, in terms of $\exp (\xi)$ in the form :

$$
u=U(\xi), \quad \xi=\alpha(x-\beta t),
$$

Substituting Eq. (5.9) into Eq. (1.1) yields an ODE:

$\beta^{2} U^{\prime \prime}-U^{\prime \prime}-15 \alpha^{2} U U^{(4)}-30 \alpha^{2} U^{\prime} U^{\prime \prime \prime}-15 \alpha^{2} U^{\prime \prime 2}-45 U^{2} U^{\prime \prime}-90 U U^{\prime 2}-\alpha^{4} U^{(6)}=0$,

Integrating (5.10) twice and using the constants of integration to be zero, we find:

$\beta^{2} U-U-15 \alpha^{2} U U^{\prime \prime}-15 U^{3}-\alpha^{4} U^{(4)}=0$,

By use of the exp-function method, we may choose the solution of (5.11) in the form:

$$
U=a_{0}+\frac{a_{1}}{1+e^{\xi}}+\frac{a_{2}}{\left(1+e^{\xi}\right)^{2}},
$$

Differentiating (5.12) with respect to $\xi$, introducing the result into Eq. (5.11), and setting the coefficients of the same power of $e^{\xi}$ equal to zero, we obtain these algebraic equations:

$$
\begin{aligned}
& -90 a_{1} a_{2} a_{0}-45 a_{1}^{2} a_{0}+\beta^{2} a_{2}-15 a_{1}^{3}-a_{1}+\beta^{2} a_{0}-45 a_{1}^{2} a_{2}-15 a_{2}^{3}-45 a_{2}^{2} a_{0}-a_{0} \\
& -15 a_{0}{ }^{3}-45 a_{1} a_{2}^{2}+\beta^{2} a_{1}-45 a_{1} a_{0}{ }^{2}-45 a_{2} a_{0}{ }^{2}=0 \\
& -90 a_{2}{ }^{2} a_{0}-90 a_{1}{ }^{2} a_{2}-90 a_{0}{ }^{3}+15 \alpha^{2} a_{0} a_{1}+30 \alpha^{2} a_{0} a_{2}-5 a_{1}+45 \alpha^{2} a_{1} a_{2}+30 \alpha^{2} a_{2}{ }^{2} \\
& -225 a_{1} a_{0}{ }^{2}+4 \beta^{2} a_{2}-180 a_{2} a_{0}{ }^{2}-270 a_{1} a_{2} a_{0}+15 \alpha^{2} a_{1}{ }^{2}-45 a_{1} a_{2}{ }^{2}+5 \beta^{2} a_{1}-180 a_{1}{ }^{2} a_{0} \\
& -45 a_{1}^{3}+\alpha^{4} a_{1}-6 a_{0}-4 a_{2}+2 \alpha^{4} a_{2}+6 \beta^{2} a_{0}=0 \\
& 15 \alpha^{2} a_{1}^{2}-10 a_{1}-60 \alpha^{2} a_{2}^{2}+15 \beta^{2} a_{0}-270 a_{1}{ }^{2} a_{0}-45 a_{2}{ }^{2} a_{0}-15 a_{0}-270 a_{2} a_{0}{ }^{2}-6 a_{2} \\
& +10 \beta^{2} a_{1}-225 a_{0}{ }^{3}+6 \beta^{2} a_{2}-45 a_{1}{ }^{2} a_{2}-30 \alpha^{2} a_{1} a_{2}-36 \alpha^{4} a_{2}-10 \alpha^{4} a_{1}-450 a_{1} a_{0}{ }^{2} \\
& -270 a_{1} a_{2} a_{0}-45 a_{1}^{3}+30 \alpha^{2} a_{0} a_{1}=0 \\
& 20 \beta^{2} a_{0}-20 a_{0}-180 a_{1}{ }^{2} a_{0}-450 a_{1} a_{0}{ }^{2}-10 a_{1}-75 \alpha^{2} a_{1} a_{2}-15 \alpha^{2} a_{1}{ }^{2}-90 a_{1} a_{2} a_{0} \\
& +66 \alpha^{4} a_{2}-180 a_{2} a_{0}{ }^{2}-15 a_{1}{ }^{3}-300 a_{0}{ }^{3}-90 \alpha^{2} a_{0} a_{2}+4 \beta^{2} a_{2}+10 \beta^{2} a_{1}-4 a_{2}=0
\end{aligned}
$$


$15 \alpha^{2} a_{1}^{2}+5 \beta^{2} a_{1}-60 \alpha^{2} a_{0} a_{2}+15 \beta^{2} a_{0}+\beta^{2} a_{2}-a_{2}-15 a_{0}-5 a_{1}-45 a_{2} a_{0}^{2}-30 \alpha^{2} a_{0} a_{1}$ $+10 \alpha^{4} a_{1}-45 a_{1}^{2} a_{0}-225 a_{1} a_{0}^{2}-225 a_{0}^{3}=0$

$6 \beta^{2} a_{0}-6 a_{0}-a_{1}-\alpha^{4} a_{1}-90 a_{0}^{3}-45 a_{1} a_{0}^{2}-15 \alpha^{2} a_{0} a_{1}=0$

$\beta^{2} a_{0}-15 a_{0}^{3}-a_{0}=0$

With the aid of Maple, the solutions of these algebraic equations are found to be:

$$
\begin{aligned}
& a_{0}=0, \quad a_{1}=2 \alpha^{2}, \\
& a_{2}=-2 \alpha^{2}, \beta=\sqrt{1+\alpha^{4}},
\end{aligned}
$$

And

$$
\begin{aligned}
& a_{0}=\left(-\frac{1}{4}+\frac{1}{60} \sqrt{105}\right) \alpha^{2}, \quad a_{1}=2 \alpha^{2}, \\
& a_{2}=-2 \alpha^{2}, \quad \beta=\sqrt{1-\frac{1}{2} \alpha^{4}-\frac{15}{2}\left(-\frac{1}{4}+\frac{1}{60} \sqrt{105}\right) \alpha^{4},}
\end{aligned}
$$

Substituting Eq. (5.14a) and Eq. (5.143b) in Eq. (5.12), we obtain two exact solutions for Eq. (1.1) of the form:

$$
u_{3}=\frac{2 \alpha^{2}}{1+e^{\left(\alpha\left(x-\sqrt{1+\alpha^{4}} t\right)\right)}}-\frac{2 \alpha^{2}}{\left(1+e^{\left(\alpha\left(x+\sqrt{1+\alpha^{4}} t\right)\right)}\right)^{2}},
$$

And

$$
\begin{gathered}
u_{4}=\left(-\frac{1}{4}+\frac{1}{60} \sqrt{105}\right) \alpha^{2}+\frac{2 \alpha^{2}}{1+e^{\left(\alpha\left(x-\sqrt{1-\frac{1}{2} \alpha^{4}-\frac{15}{2}\left(-\frac{1}{4}+\frac{1}{60} \sqrt{105}\right) \alpha^{4} t}\right)\right)}} \\
-\frac{2 \alpha^{2}}{\left(1+e^{\left.\left(\alpha\left(x-\sqrt{1-\frac{1}{2} \alpha^{4}-\frac{15}{2}\left(-\frac{1}{4}+\frac{1}{60} \sqrt{105}\right) \alpha^{4} t}\right)\right)\right)^{2}},\right.}
\end{gathered}
$$

\subsection{Application of rational hyperbolic functions methods}

We next substitute the rational hyperbolic method (4.1) for $n=1$ and $f(\mu \xi)=\sinh (\mu \xi)$ into Eq.(5.3) and Collect the coefficients of the same power of resulting hyperbolic function equal to zero, the following algebraic system will be obtained:

$$
\begin{aligned}
& c^{2} a_{0}-a_{0}-30 \mu^{2} a_{1}{ }^{2} a_{0}{ }^{2}-15 a_{0}^{3}+24 b_{0} \mu^{4} a_{1}^{3}+30 \mu^{2} a_{0} b_{0} a_{1}-24 a_{1}^{4} \mu^{4} a_{0}-8 a_{1}^{2} \mu^{4} a_{0} \\
& +8 b_{0} \mu^{4} a_{1}=0 \\
& -4 a_{0} a_{1}-b_{0}-45 a_{0}{ }^{2} b_{0}+20 a_{1}^{3} \mu^{4} a_{0}+30 \mu^{2} b_{0}{ }^{2} a_{1}+4 c^{2} a_{0} a_{1}-30 \mu^{2} a_{1}{ }^{3} a_{0}{ }^{2} \\
& -30 a_{0}{ }^{3} a_{1}+c^{2} b_{0}-20 b_{0} \mu^{4} a_{1}{ }^{2}+a_{1} \mu^{4} a_{0}-15 \mu^{2} a_{0} b_{0}-b_{0} \mu^{4}+15 \mu^{2} a_{0}{ }^{2} a_{1}=0
\end{aligned}
$$




$$
\begin{aligned}
& -11 a_{1}^{2} \mu^{4} a_{0}-45 a_{0} b_{0}{ }^{2}-20 a_{1}^{4} \mu^{4} a_{0}+4 c^{2} b_{0} a_{1}+30 \mu^{2} b_{0}{ }^{2} a_{1}^{2}-30 \mu^{2} b_{0} a_{1}^{3} a_{0} \\
& -90 a_{0}{ }^{2} b_{0} a_{1}-6 a_{1}^{2} a_{0}+6 c^{2} a_{1}^{2} a_{0}-15 \mu^{2} b_{0}{ }^{2}-4 b_{0} a_{1}+15 \mu^{2} a_{0} b_{0} a_{1}+11 b_{0} \mu^{4} a_{1} \\
& +20 b_{0} \mu^{4} a_{1}^{3}-15 a_{0}{ }^{3} a_{1}^{2}=0 \\
& -15 \mu^{2} a_{1}^{3} a_{0}^{2}-11 b_{0} \mu^{4} a_{1}^{2}+6 c^{2} b_{0} a_{1}^{2}+4 c^{2} a_{0} a_{1}^{3}-45 a_{0}{ }^{2} b_{0} a_{1}^{2}-15 b_{0}^{3} \\
& +11 a_{1}^{3} \mu^{4} a_{0}-90 a_{0} b_{0}{ }^{2} a_{1}-4 a_{0} a_{1}^{3}-6 b_{0} a_{1}^{2}+15 \mu^{2} b_{0} a_{1}^{2} a_{0}=0 \\
& b_{0} \mu^{4} a_{1}^{3}-a_{1}{ }^{3} \mu^{4} a_{0}-4 b_{0} a_{1}^{3}-30 b_{0}{ }^{3} a_{1}-15 \mu^{2} b_{0} a_{1}^{3} a_{0}+15 \mu^{2} b_{0}{ }^{2} a_{1}^{2} \\
& -a_{0} a_{1}^{4}+4 c^{2} b_{0} a_{1}^{3}+c^{2} a_{0} a_{1}^{4}-45 a_{0} b_{0}{ }^{2} a_{1}^{2}=0 \\
& -b_{0} a_{1}^{4}+c^{2} b_{0} a_{1}^{4}-15 b_{0}{ }^{3} a_{1}^{2}=0
\end{aligned}
$$

Solving the above system, we the following results will be obtained:

$$
\begin{aligned}
& a_{0}=\mu^{2}, \quad a_{1}=I, \\
& b_{0}=0, c=\sqrt{\mu^{4}+1},
\end{aligned}
$$

And

$$
\begin{aligned}
& a_{0}=\left(\frac{3}{4}-\frac{1}{60} \sqrt{105}\right) \mu^{2}, \quad a_{1}=I, \\
& b_{0}=\frac{\frac{1}{2} I \mu^{2}\left(\frac{3}{4}-\frac{1}{60} \sqrt{105}\right)}{\frac{13}{14}-\frac{1}{4} \sqrt{105}}, c=\sqrt{1-\frac{15}{2} \mu^{4}\left(\frac{3}{4}-\frac{1}{60} \sqrt{105}\right)+7 \mu^{4}},
\end{aligned}
$$

Substituting Eq. (5.17a) and Eq. (5.17b) in Eq. (4.1) along with $n=2$, and $f(\mu \xi)=\sinh (\mu \xi)$, we obtain two exact solutions of Eq. (1.1):

$$
u_{5}=\frac{\mu^{2}}{\left(1+I \sinh \left(\mu\left(x-\sqrt{\mu^{4}+1} t\right)\right)\right)},
$$

And

$$
\begin{aligned}
& u_{6}=\frac{\left(\frac{3}{4}-\frac{1}{60} \sqrt{105}\right) \mu^{2}}{1+I \sinh \left(\mu\left(x-\sqrt{1-\frac{15}{2} \mu^{4}\left(\frac{3}{4}-\frac{1}{60} \sqrt{105}\right)+7 \mu^{4} t}\right)\right)} \\
& +\frac{\frac{1}{2} I \mu^{2}\left(\frac{3}{4}-\frac{1}{60} \sqrt{105}\right)}{\frac{13}{14}-\frac{1}{4} \sqrt{105}} \sinh \left(\mu\left(x-\sqrt{1-\frac{15}{2} \mu^{4}\left(\frac{3}{4}-\frac{1}{60} \sqrt{105}\right)+7 \mu^{4} t}\right)\right) \\
& 1+I \sinh \left(\mu\left(x-\sqrt{1-\frac{15}{2} \mu^{4}\left(\frac{3}{4}-\frac{1}{60} \sqrt{105}\right)+7 \mu^{4} t}\right)\right)
\end{aligned}
$$


If we choose $n=2$ and $f(\mu \xi)=\sinh (\mu \xi)$, and consider the rational of hyperbolic method solution of the form (4.1) and insert them into Eq.(5.3), and Collect the coefficients of the same power of resulting hyperbolic function equal to zero, the following algebraic system will be resulted:

$$
\begin{aligned}
& -24 a_{1}^{2} \mu^{4} a_{0}+c^{2} a_{0}+30 \mu^{2} a_{1} a_{0}^{2}-15 a_{0}^{3}-30 \mu^{2} a_{0} b_{0}-8 b_{0} \mu^{4}+24 b_{0} \mu^{4} a_{1} \\
& +8 a_{1} \mu^{4} a_{0}-a_{0}=0 \\
& 60 \mu^{2} a_{1} a_{0}^{2}+c^{2} b_{0}+240 a_{1}^{3} \mu^{4} a_{0}+90 \mu^{2} a_{0} b_{0} a_{1}-4 a_{1} a_{0}-45 a_{0}^{2} b_{0}-b_{0}+200 b_{0} \mu^{4} a_{1} \\
& -30 a_{0}{ }^{3} a_{1}-240 b_{0} \mu^{4} a_{1}{ }^{2}-30 \mu^{2} b_{0}{ }^{2}-60 \mu^{2} a_{0} b_{0}+4 c^{2} a_{0} a_{1}-200 a_{1}{ }^{2} \mu^{4} a_{0}-60 \mu^{2} a_{1}{ }^{2} a_{0}{ }^{2} \\
& +16 a_{1} \mu^{4} a_{0}-16 b_{0} \mu^{4}=0 \\
& 440 a_{1}^{3} \mu^{4} a_{0}-90 a_{0}{ }^{2} b_{0} a_{1}-60 \mu^{2} b_{0}{ }^{2}-45 a_{0} b_{0}{ }^{2}+6 c^{2} a_{1}{ }^{2} a_{0}+60 \mu^{2} a_{0} b_{0} a_{1}-120 a_{1}{ }^{4} \mu^{4} a_{0} \\
& -4 b_{0} a_{1}-90 \mu^{2} a_{1}^{3} a_{0}^{2}-6 a_{1}^{2} a_{0}-176 a_{1}^{2} \mu^{4} a_{0}+60 \mu^{2} b_{0}^{2} a_{1}+176 b_{0} \mu^{4} a_{1}+4 c^{2} b_{0} a_{1} \\
& -440 b_{0} \mu^{4} a_{1}^{2}+120 b_{0} \mu^{4} a_{1}^{3}-15 a_{0}^{3} a_{1}^{2}+30 \mu^{2} b_{0} a_{1}^{2} a_{0}=0 \\
& -4 a_{0} a_{1}^{3}-90 \mu^{2} b_{0} a_{1}^{3} a_{0}+176 a_{1}^{3} \mu^{4} a_{0}-45 a_{0}{ }^{2} b_{0} a_{1}^{2}+6 c^{2} b_{0} a_{1}{ }^{2}-15 b_{0}{ }^{3}+4 c^{2} a_{0} a_{1}^{3} \\
& -60 \mu^{2} a_{1}^{3} a_{0}^{2}+90 \mu^{2} b_{0}^{2} a_{1}^{2}-6 b_{0} a_{1}^{2}-176 b_{0} \mu^{4} a_{1}^{2}-90 a_{0} b_{0}^{2} a_{1}+60 \mu^{2} b_{0} a_{1}^{2} a_{0} \\
& -120 a_{1}^{4} \mu^{4} a_{0}+120 b_{0} \mu^{4} a_{1}^{3}=0 \\
& c^{2} a_{0} a_{1}^{4}-4 b_{0} a_{1}^{3}+16 b_{0} \mu^{4} a_{1}^{3}-45 a_{0} b_{0}{ }^{2} a_{1}{ }^{2}-60 \mu^{2} b_{0} a_{1}{ }^{3} a_{0}-16 a_{1}{ }^{4} \mu^{4} a_{0} \\
& +60 \mu^{2} b_{0}{ }^{2} a_{1}^{2}+4 c^{2} b_{0} a_{1}^{3}-a_{0} a_{1}^{4}-30 b_{0}^{3} a_{1}=0 \\
& -b_{0} a_{1}^{4}+c^{2} b_{0} a_{1}^{4}-15 b_{0}^{3} a_{1}^{2}=0
\end{aligned}
$$

With the aid of Maple, the solutions of these algebraic equations are found to be:

$$
\begin{aligned}
& a_{0}=2 \mu^{2}, \quad a_{1}=1, \\
& b_{0}=0, c=\sqrt{16 \mu^{4}+1},
\end{aligned}
$$

And

$$
\begin{aligned}
& a_{0}=2\left(\frac{1}{2}-\frac{1}{30} \sqrt{105}\right) \mu^{2}, \quad a_{1}=1, \\
& b_{0}=\frac{4}{15} \frac{\mu^{2} \sqrt{105}}{\frac{7}{2}-\frac{1}{2} \sqrt{105}}, c=\sqrt{1-60 \mu^{4}\left(\frac{1}{2}-\frac{1}{30} \sqrt{105}\right)+52 \mu^{4}},
\end{aligned}
$$

Substituting Eq. (5.20a) and Eq. (5.20b) in Eq. (4.1) along with $n=2$, and $f(\mu \xi)=\sinh (\mu \xi)$,we obtain another two exact solutions of Eq. (1.1): 


$$
u_{7}=\frac{2 \mu^{2}}{1+I \sinh ^{2}\left(\mu\left(x-\sqrt{16 \mu^{4}+1} t\right)\right)},
$$

And

$$
\begin{aligned}
& u_{8}= \frac{2\left(\frac{1}{2}-\frac{1}{30} \sqrt{105}\right) \mu^{2}}{1+\sinh ^{2}\left(\mu\left(x-\sqrt{1-60 \mu^{4}\left(\frac{1}{2}-\frac{1}{30} \sqrt{105}\right)+52 \mu^{4} t}\right)\right)}+ \\
& \frac{\left(\frac{4}{15} \frac{\mu^{2} \sqrt{105}}{2}-\frac{1}{2} \sqrt{105}\right.}{10} \sinh ^{2}\left(\mu\left(x-\sqrt{1-60 \mu^{4}\left(\frac{1}{2}-\frac{1}{30} \sqrt{105}\right)+52 \mu^{4} t}\right)\right) \\
& 1+\sinh ^{2}\left(\mu\left(x-\sqrt{1-60 \mu^{4}\left(\frac{1}{2}-\frac{1}{30} \sqrt{105}\right)+52 \mu^{4} t}\right)\right)
\end{aligned}
$$

We now consider the rational hyperbolic function of the form (4.1) with $n=2$ and $f(\mu \xi)=\tanh (\mu \xi)$ :

$U(\xi)=\frac{a_{0}+b_{0} \tanh ^{2}(\mu \xi)}{1+a_{1} \tanh ^{2}(\mu \xi)}$

Substituting Eq.(5.22) into Eq.(4.1) and Collecting the coefficients of the same power of resulting hyperbolic function equal to zero, the following algebraic system will be resulted:

$$
\begin{aligned}
& -24 a_{1}^{2} \mu^{4} a_{0}-a_{0}-15 a_{0}^{3}-30 \mu^{2} a_{0} b_{0}+c^{2} a_{0}+30 \mu^{2} a_{1} a_{0}^{2}-16 a_{1} \mu^{4} a_{0} \\
& +16 b_{0} \mu^{4}+24 b_{0} \mu^{4} a_{1}=0 \\
& c^{2} b_{0}+136 a_{1} \mu^{4} a_{0}-120 \mu^{2} a_{1} a_{0}^{2}+120 \mu^{2} a_{0} b_{0}-60 \mu^{2} a_{1}^{2} a_{0}^{2}+90 \mu^{2} a_{0} b_{0} a_{1} \\
& -30 \mu^{2} b_{0}{ }^{2}-b_{0}-240 a_{1}{ }^{2} \mu^{4} b_{0}-30 a_{0}{ }^{3} a_{1}-4 a_{1} a_{0}-45 a_{0}{ }^{2} b_{0}+240 a_{1}{ }^{3} \mu^{4} a_{0} \\
& +4 c^{2} a_{0} a_{1}-136 b_{0} \mu^{4}+400 a_{1}^{2} \mu^{4} a_{0}-400 b_{0} \mu^{4} a_{1}=0 \\
& 6 c^{2} a_{1}^{2} a_{0}-6 a_{1}^{2} a_{0}-4 b_{0} a_{1}-1016 a_{1}^{2} \mu^{4} a_{0}-90 \mu^{2} a_{0} b_{0}-45 a_{0} b_{0}^{2}+120 a_{1}^{3} \mu^{4} b_{0} \\
& +30 \mu^{2} b_{0} a_{1}{ }^{2} a_{0}-90 a_{0}{ }^{2} b_{0} a_{1}-880 a_{1}{ }^{3} \mu^{4} a_{0}+120 \mu^{2} b_{0}{ }^{2}-120 a_{1}{ }^{4} \mu^{4} a_{0}+60 \mu^{2} b_{0}{ }^{2} a_{1} \\
& +4 c^{2} b_{0} a_{1}-240 a_{1} \mu^{4} a_{0}-15 a_{0}{ }^{3} a_{1}^{2}+90 \mu^{2} a_{1} a_{0}{ }^{2}+1016 b_{0} \mu^{4} a_{1}-90 \mu^{2} a_{1}{ }^{3} a_{0}{ }^{2} \\
& +880 a_{1}^{2} \mu^{4} b_{0}-120 \mu^{2} a_{0} b_{0} a_{1}+240 b_{0} \mu^{4}=0 \\
& 60 \mu^{2} b a_{1}^{2} a_{0}^{2}-90 \mu^{2} b_{0} a_{1}^{3} a_{0}+6 c^{2} b_{0} a_{1}^{2}-120 \mu^{2} b_{0} a_{1}^{2} a_{0}+1016 a_{1}^{3} \mu^{3} a_{0}-45 a_{0}^{2} b_{0} a_{1}^{2} \\
& -15 b_{0}{ }^{3}+30 \mu^{2} a_{0} b_{0} a_{1}+240 a_{1}^{4} \mu^{4} a_{0}+90 \mu^{2} b_{0}{ }^{2} a_{1}{ }^{2}-120 b_{0} \mu^{4}-6 a_{1}^{2} b_{0}+880 a_{1}^{2} \mu^{4} a_{0} \\
& -4 a_{1}^{3} a_{0}+4 c^{2} a_{0} a_{1}^{3}-90 a_{0} b_{0}{ }^{2} a_{1}+120 \mu^{2} a_{1}{ }^{3} a_{0}{ }^{2}+120 a_{1} \mu^{4} a_{0}-240 a_{1}^{3} \mu^{4} b_{0}-90 \mu^{2} b_{0}{ }^{2} \\
& -880 b_{0} \mu^{4} a_{1}-1016 a_{1}^{2} \mu^{4} b_{0}=0
\end{aligned}
$$




$$
\begin{aligned}
& 4 c^{2} b_{0} a_{1}^{3}+90 \mu^{2} b_{0} a_{1}^{2} a_{0}-136 a_{1}^{4} \mu^{4} a_{0}-60 \mu^{2} b_{0}{ }^{2} a_{1}+136 a_{1}^{3} \mu^{4} b_{0}-4 a_{1}^{3} b_{0} \\
& -120 \mu^{2} b_{0}{ }^{2} a_{1}^{2}-45 a_{0} b_{0}{ }^{2} a_{1}^{2}-240 a_{1}^{2} \mu^{4} a_{1}-400 a_{1}^{3} \mu^{4} a_{0}+c^{2} a_{0} a_{1}^{4}-30 b_{0}{ }^{3} a_{1} \\
& +120 \mu^{2} b_{0} a_{1}^{3} a_{0}+400 a_{1}^{2} \mu^{4} b_{0}-30 \mu^{2} a_{1}^{3} a_{0}{ }^{2}-a_{1}{ }^{4} a_{0}=0 \\
& 30 \mu^{2} b_{0}{ }^{2} a_{1}^{2}-b_{0} a_{1}^{4}-30 \mu^{2} b_{0} a_{1}^{3} a_{0}-16 a_{1}^{3} \mu^{4} b_{0}-24 a_{1}^{2} \mu^{4} b_{0}-15 b_{0}{ }^{3} a_{1}^{2} \\
& +16 a_{1}^{4} \mu^{4} a_{0}+24 a_{1}^{3} \mu^{4} a_{0}+c^{2} b_{0} a_{1}^{4}=0
\end{aligned}
$$

With the aid of Maple, the solutions of these algebraic equations are found to be:

$$
\begin{aligned}
& a_{0}=2 \mu^{2}, \quad a_{1}=0, \\
& b_{0}=-2 \mu^{2}, c=\sqrt{16 \mu^{4}+1},
\end{aligned}
$$

\section{And}

$$
\begin{aligned}
& a_{0}=2\left(\frac{1}{2}-\frac{1}{30} \sqrt{105}\right) \mu^{2}, \quad a_{1}=0 \\
& b_{0}=-2 \mu^{2}, c=\sqrt{1-60 \mu^{4}\left(\frac{1}{2}-\frac{1}{30} \sqrt{105}\right)+52 \mu^{4}}
\end{aligned}
$$

Substituting Eq. (5.24a) and Eq. (5.24b) in Eq. (5.22),we obtain another two exact solutions of Eq. (1.1):

$u_{7}=2 \mu^{2}-2 \mu^{2} \tanh ^{2}\left(\mu\left(x-\sqrt{16 \mu^{4}+1} t\right)\right)$,

And

$u_{7}=2\left(\frac{1}{2}-\frac{1}{30} \sqrt{105}\right) \mu^{2}-2 \mu^{2} \tanh ^{2}\left(\mu\left(x-\sqrt{1-60 \mu^{4}\left(\frac{1}{2}-\frac{1}{30} \sqrt{105}\right)+52 \mu^{4} t}\right)\right)$,

\section{5- Conclusions}

In summary, we have applied the sin-cosine method along with rational exponential function and rational hyperbolic function method to obtain travelling wave solution for the sixth-order Boussinesq equation. In fact, the present methods are readily applicable to a large variety of such nonlinear equations. It is shown that The hyperbolic ansatze handled Gardner equation effectively and the sin-cosine method and the rational exponential function method are powerful and straightforward solution method to find closed-form, periodic and nonperiodic analytical expressions for travelling waves of nonlinear wave and evolution equations.

\section{References}

[1] G. Eilenberger, Solitons (Springer-Verlag, Berlin, 1983).

[2] G. Whitham, Linear and Nonlinear Waves (Wiley, New York, 1974).

[3] P. Gray and S. Scott, Chemical Oscillations and Instabilities (Clarendon, Oxford, 1990).

[4] A. Hasegawa, Plasma Instabilities and Nonlinear Effects (Springer-Verlag, Berlin, 1975).

[5] C. S. Gardner, J. M. Green, D. Kruskal and R. M. Miura, Phys. Rev. Lett. 19, (1967), 1095-1105

[6] M.J. Ablowitz, Segur, H. Solitons and inverse scattering transform .SIAM. Philadelphia (1981) 
[7] R .Hirota, Direct method of finding exact solutions of nonlinear evolution equations. R. Bullough, P.Caudrey (Eds.), Backlund transformations, Springer, Berlin (1980), 1157-1175

[8] M. Wadati, H. Sanuki, Konno K. Relationships among inverse method, Backlund transformation and an infinite number of conservation laws. Prog Theor Phys 53,(1975);419-36.

[9] M.J. Ablowitz, P.A. Clarkson, Solitons, Nonlinear Evolution Equations and Inverse Scattering, Cambridge Univ. Press, Cambridge, 1991.

[10] A. Coely, et al. (Eds.), Bäcklund and Darboux Transformations, American Mathematical Society, Providence, RI, 2001.

[11] V. B. Matveev and M. A. Salle, Darboux Transformations and Solitons (Springer-Verlag, Berlin, 1991).

[12] W. Malfliet, Solitary wave solutions of nonlinear wave equations, Amer. J. Phys. 60 (1992) 650-654.

[13] W. Malfliet, W. Hereman, The tanh method: I. Exact solutions of nonlinear evolution and wave equations, Physica. Scripta 54 (1996) 563-568.

[14] D.D. Ganji, M. Abdollahzadeh, Exact travelling solutions for the Lax's seventh-order KdV equation by sech method and rational exp-function method, Applied Mathematics and Computation 206 (2008) 438-444

[15] Engui Fan, Extended tanh-function method and its applications to nonlinear equations, Physics Letters A 277 (2000) 212-218.

[16] Ji-Huan $\mathrm{He}$, Xu-Hong $\mathrm{Wu}$, Exp-function method for nonlinear wave equations, Chaos, Solitons and Fractals 30 (2006) 700-708.

[17] Ji-Huan He, M.A. Abdou, New periodic solutions for nonlinear evolution equations using Exp-function method, Chaos, Solitons and Fractals 34 (2007) 1421-1429.

[18] Xu-Hong (Benn)Wu , Ji-Huan He, EXP-function method and its application to nonlinear equations, Chaos, Solitons and Fractals 38 (2008) 903-910

[19] Z.Z. Ganji, D.D. Ganji, H. Bararnia, Approximate general and explicit solutions of nonlinear BBMB equations by Exp-Function method, Applied Mathematical Modelling (2008),in press, doi:10.1016/j.apm.2008.03.005

[20] Abdul-Majid Wazwaz: The sine-cosine method for obtaining solutions with compact and noncompact structures. Applied Mathematics and Computation 159 (2004) 559-576

[21] Abdul-Majid Wazwaz , M.A. Helal , Nonlinear variants of the BBM equation with compact and noncompact physical structures, Chaos, Solitons and Fractals 26 (2005) 767-776

[22] Wazwaz, A.M.: A sine-cosine method for handling nonlinear wave equations. Mathematical And Computer Modeling 40(2004),499-508

[23] Shikuo Liu , Zuntao Fu , Shida Liu , Qiang Zhao, Jacobi elliptic function expansion method and periodic wave solutions of nonlinear wave equations, Physics Letters A 289 (2001) 69-74.

[24] Zuntao Fu , Shikuo Liu , Shida Liu , Qiang Zhao, New Jacobi elliptic function expansion and new periodic solutions of nonlinear wave equations, Physics Letters A 290 (2001) 72-76.

[25] E.J. Parkes , B.R. Duffy, P.C. Abbott, The Jacobi elliptic-function method for finding periodicwave solutions to nonlinear evolution equations, Physics Letters A 295 (2002) 280-286.

[26] D.D. Ganji, M. Abdollahzadeh, Exact traveling solutions of some nonlinear evolution equation by (G'/G)-expansion method, JOURNAL OF MATHEMATICAL PHYSICS 50, 1 (2009)

[27] M. Abdollahzadeh, M. Ghanbarpour, M. Hosseini , H. Ebrahimnejad .Exact travelling solutions for fifth order Caudrey-Dodd-Gibbon equation, International Journal of Applied Mathematics and Computation, Accepted Articles : In Press

[28] P. J. Olver, Application of Lie Group to Differential Equation (Springer, New York, 1986). 
[29]A. Sadighi and D.D. Ganji, Exact solutions of nonlinear diffusion equations by variational iteration method, Computers and Mathematics with Applications 54 (2007) 1112-1121

[30]D.D. Ganji and M. Rafei, Solitary wave solutions for a generalized Hirota-Satsuma coupled KdV equation by homotopy perturbation method, Physics Letters A 356 (2006) 131-137

[31]D.D. Ganji, The application of He's homotopy perturbation method to nonlinear equations arising in heat transfer, Physics Letters A, 355, (2006) 337-341.

[32] D.D. Ganji, G.A. Afrouzi, R.A. Talarposhti, Application of variational iteration method and homotopy-perturbation method for nonlinear heat diffusion and heat transfer equations ,

Physics Letters A 368 (2007) 450-457

[33] Engui Fan, Hongqing Zhang.: A note on the homogeneous balance method. Physics Letters A 246 (1998) 403406

[34] Lixia Wang, Jiangbo Zhou, Lihong Ren: The Exact Solitary Wave Solutions for a Family of BBM Equation.International Journal of Nonlinear Science, Vol. 1 (2006) No. 1, pp. 58-64

[35] Yan Z. Constructing exact solutions for two-dimensional nonlinear dispersion Boussinesq equation. II: Solitary pattern solutions.Chaos, Solitons \& Fractals 2003;18(4):869-80.

[36] Wazwaz AM. Two reliable methods for solving variants of the KdV equation with compact and noncompact structures. Chaos,Solitons \& Fractals 2006;28(2):454-62.

[37] M Wazwaz A. New compactons, solitons and periodic solutions for nonlinear variants of the KdV and the KP equations. Chaos,Solitons \& Fractals 2004;22(1):249-60.

[38] M Wazwaz A. Exact and explicit travelling wave solutions for the nonlinear DrinfeldSokolov system. Commun Nonlin SciNumer Simul 2006;11:311-25.

[39] Abdul-Majid Wazwaz, Multiple-soliton solutions for the ninth-order KdV equation and sixth-order Boussinesq equation, Applied Mathematics and Computation 203 (2008) 277-283

[40] Sheng Zhang, Application of Exp-function method to a KdV equation with variable coefficients, Physics Letters A 365 (2007) 448-453.

[41] Sheng Zhang, Application of Exp-function method to high-dimensional nonlinear evolution equations, Chaos, Solitons and Fractals 38 (2008) 270-276.

[42] A. Ebaid, Exact solitary wave solutions for some nonlinear evolution equations via Expfunction method, Physics Letters A 365 (2007) 213-219.

[43] E. Yusufoglu, A. Bekir, Symbolic computation and new families of exact travelling solutions for the Kawahara and modified Kawahara equations, Computers and Mathematics with Applications 55 (2008) 1113-1121.

[44] Abdul-Majid Wazwaz, New solitary wave solutions to the modified forms of DegasperisProcesi. and Camassa-Holm equations, Applied Mathematics and Computation 186 (2007) 130-141.

[45] Abdul-Majid Wazwaz, New solitons and kink solutions for the Gardner equation, Communications in Nonlinear Science and Numerical Simulation 12 (2007) 1395-1404. 